\title{
Study of Uniaxial Tensile Properties of Hexagonal Boron Nitride Nanoribbons
}

\author{
Ratul Paul, Tamanna Tasnim, Rajkumar Dhar, Satyajit Mojumder, Sourav Saha, Mohammad Abdul Motalab* \\ Department of Mechanical Engineering \\ Bangladesh University of Engineering and Technology, Dhaka-1000 \\ *Email: mtipuz@yahoo.com, Telephone: +8801779198595,+880-2-9665636, 9665650 ext:7230
}

\begin{abstract}
Uniaxial tensile properties of hexagonal boron nitride nanoribbons and dependence of these properties on temperature, strain rate, and the inclusion of vacancy defects have been explored with molecular dynamics simulations using Tersoff potential. The ultimate tensile strength of pristine hexagonal boron nitride nanoribbon of $26 \mathrm{~nm} \times 5 \mathrm{~nm}$ with armchair chirality is found to be $100.5 \mathrm{GPa}$. The ultimate tensile strength and strain have been found decreasing with increasing the temperature while an opposite trend has been observed for increasing the strain rate. Furthermore, the vacancy defects reduce ultimate tensile strength and strain where the effect of bi-vacancy is clearly dominating over point vacancy.
\end{abstract}

Keywords-Molecular dynamics simulation, hexagonal boron nitride nanoribbons, uniaxial tensile test.

\section{INTRODUCTION}

The hexagonal boron nitride nanostructures have received research interest due to their supreme electrical, chemical and mechanical properties. In some aspects, $\mathrm{hBN}$ holds advantages over its analogue graphene like electrical insulation with large band gap, [1] thermal and chemical stabilities, high thermal conductivities [2], [3] and comparable mechanical properties. [4] These remarkable properties have made hBN nanostructures to have many potential applications in engineering fields. [5]

We have studied hexagonal boron nitride nanoribbons (hBNNRs) in this paper as it is less explored than other nanostructures. As hBNNRs possess high tensile strength, [6], [7] it is possible to enhance tensile strength of polymer matrix by using them for structural reinforcement. [8], [9]. The advantage of using nanoribbons over other nanostructures lies in its active chemical edges. These active edges create strong chemical bonding between filler and matrix composites. Moreover, it is also found that mechanical properties of BNNRs can be improved by narrowing width. [10] Thus hBNNRs poses prospect in reinforcement applications of matrix composites.

Fabrication process of hBNNRs is still challenging. One way to accomplish this is by unfolding hexagonal Boron Nitride nanotubes (hBNNTs) [11]. Chemical vapor deposition (CVD) technique also shows potential in hBNNRs synthesis [12]. As seen in the case of other nanostructures, CVD incurs unavoidable defects in nanostructures. These defects impose impacts on electrical and mechanical properties of nanomaterials. [13]-[15] Sinitsii et al. [16] demonstrated potassium induced splitting of BNNTs to fabricate BNNRs and there the presence of defects was also evident. Therefore, taking into account of the inevitability of structural defects, we have studied the effect of various defects on the properties of hBNNRs.

Defects in the form of vacancies like point vacancy and edge vacancy have been presented in this paper. Mortazavi et al. [17] and Khan et al. [18] showed the effects of randomly distributed defects on the thermal conductivity and tensile response of single layer graphene sheets. Using molecular dynamics simulation with Tersoff potential, we have analyzed the change in tensile strength of hBNNRs with the inclusion of vacancies. It is observed that with the increasing concentration of vacancies, tensile strength decreases. We have also shown the effect of temperature and strain rate on the tensile strength of hBNNRs and the modified properties have been compared to that of pristine hBNNRs.

\section{SIMULATION MODEL}

In this study, molecular dynamics simulations have been carried out using LAMMPS [19] (Large-scale Atomic/Molecular Massively Parallel Simulator) for 


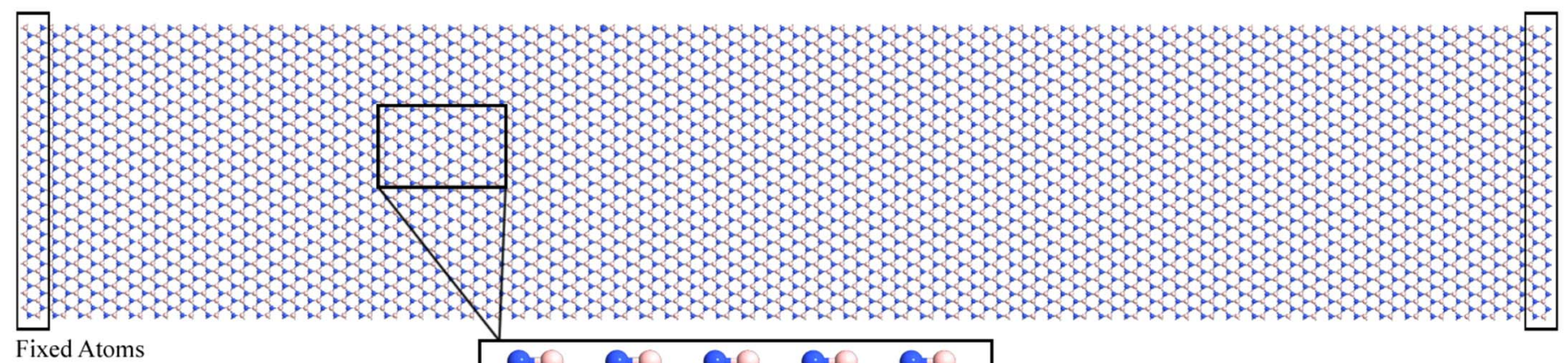

Fixed Atoms
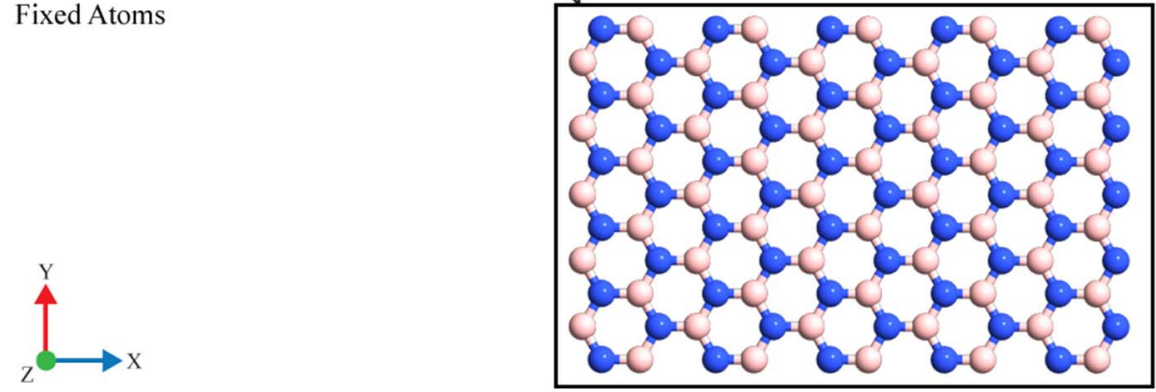

Boron Atoms

Nitrogen Atoms

Fig. 1: Structure of $26 \mathrm{~nm}$ x $5 \mathrm{~nm}$ hBNNR with magnified view of 5 x 5 cell. Light pink atoms represent boron where blue atoms represent nitrogen.

calculating the tensile properties of hBNNR. To define the interatomic interactions between boron and nitrogen atoms in the molecular dynamics simulations, we used Tersoff potential with parameters obtained by Lindsay and Broido. [20] For our study, we used a hBNNR structure of $26 \mathrm{~nm} \times 5 \mathrm{~nm}$ with armchair chirality containing 4800 atoms. In the modeling of tensile strength, we fix four rows of atoms at the two ends of the hBNNR in longitudinal direction in which uniaxial strain has been applied to prevent atoms from sublimating [21] as shown in Fig. 1.

Point vacancy was introduced in the structure by the removal of a randomly chosen atom from the geometry while the bi-vacancy was created by introducing two consecutive point vacancies or by simply removing a pair of bonding atoms [22], [23]. The defects are illustrated in Fig. 2. The thickness of multilayer hBNNR was assumed to be $0.33 \mathrm{~nm}$. [11], [21], [24] Hence periodic boundary conditions have been applied along the $\mathrm{X}, \mathrm{Y}$ and $\mathrm{Z}$ axis keeping a $4 \mathrm{~nm}$ gap in the simulation box of the hBNNR along the $\mathrm{Z}$ axis during the simulation process to ensure no interactions take place between the hBNNR in the simulation domain and its image. [23]

The equations of motion were solved using the VelocityVerlet algorithm [25] with a time step of $0.001 \mathrm{ps}$. The initial structure was first relaxed by conjugate gradient algorithm and then equilibrated using constant pressure and constant pressure ensemble (NPT) by means of Nose'-Hoover barostat and thermostat method for 50 ps. Strain has been applied in the structure along the $\mathrm{x}$ direction while keeping the $\mathrm{Y}$ and $\mathrm{Z}$ boundaries in zero stress using NPT ensemble. We varied the strain applied to the $\mathrm{x}$ direction and virial stress were calculated at each strain level to obtain the stress-strain response of the structure using the following formula.

$$
\sigma(r)=\frac{1}{V} \sum_{i}\left[\left(-m_{i} \stackrel{\circ}{\mathrm{u}}_{i} \otimes \stackrel{\circ}{\mathrm{u}}_{i}+\frac{1}{2} \sum_{j \neq i} r_{i j} \otimes f_{i j}\right.\right.
$$

where, $\sigma$ is the virial stress, $\mathrm{V}$ is the volume of the stanene atom, the summation is over all the atoms occupying the total volume, $m_{i}$ is the mass of atom $\stackrel{\circ}{i}_{i}$ is the time derivative which indicates the displacement of atom with respect to a reference position, $r_{i j}$ is the position vector of atom, $\otimes$ is the cross product, and $f_{i j}$ is the interatomic force applied on atom $i$ by atom $j$.

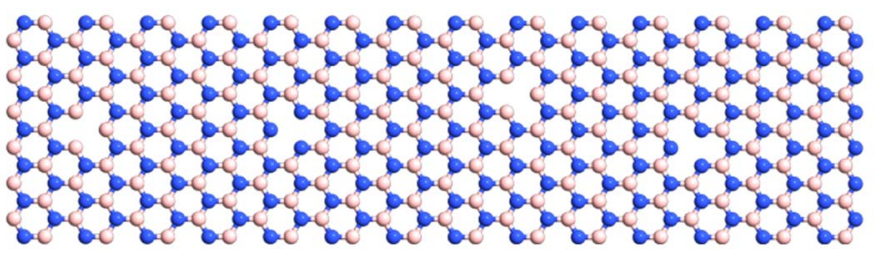

Point Vacancy

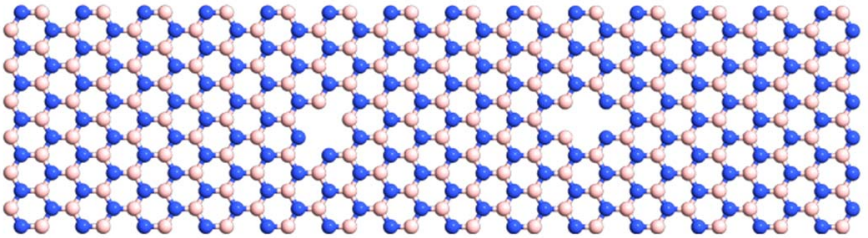

Bi-vacancy

Fig. 2: Point vacancy and Bi-vacancy in armchair hBNNR structure. A total number of four atoms are absent in both structures. 


\section{SimULATION RESULTS}

The stress- strain curves recorded for pristine $26 \mathrm{~nm} \times 5 \mathrm{~nm}$ armchair hBNNR at $100 \mathrm{~K}, 200 \mathrm{~K}, 300 \mathrm{~K}, 400 \mathrm{~K}$ and $500 \mathrm{~K}$ are shown in Fig. 3. All the simulations were conducted at a constant strain rate of $1 \times 10^{9} \mathrm{~s}^{-1}$. It is clear that the ultimate uniaxial tensile strength and strain decrease gradually with rising temperature.

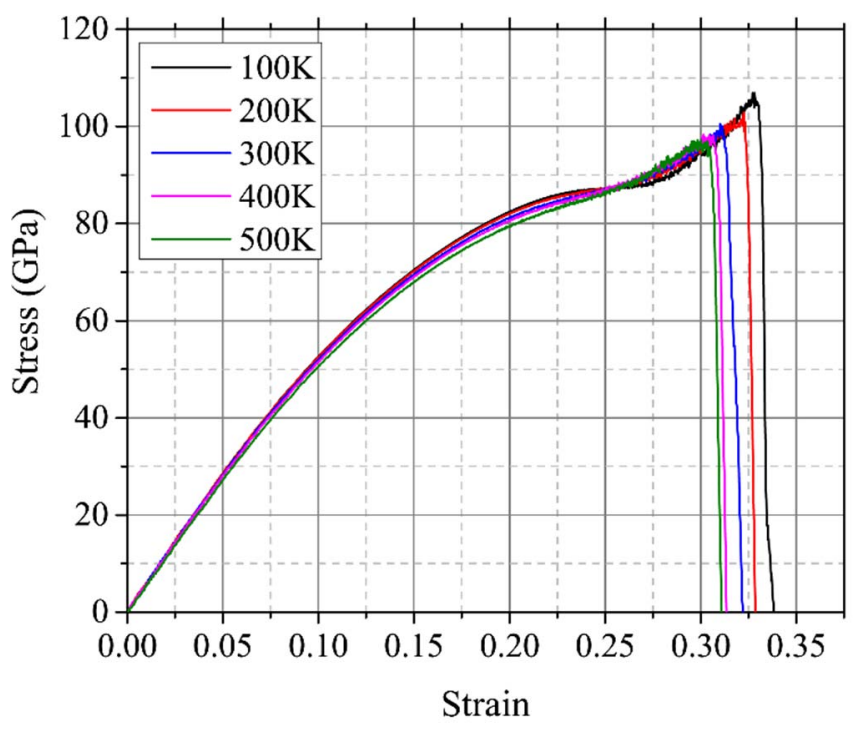

Fig. 3: Stress-strain curves of $26 \mathrm{~nm} \times 5 \mathrm{~nm}$ armchair hBNNR at different temperature at a constant strain rate of $1 \times 10^{9} \mathrm{~s}^{-1}$

The ultimate uniaxial tensile strength and strain relations with temperature for pristine hBNNR are shown in Fig. 4 and Fig. 5.

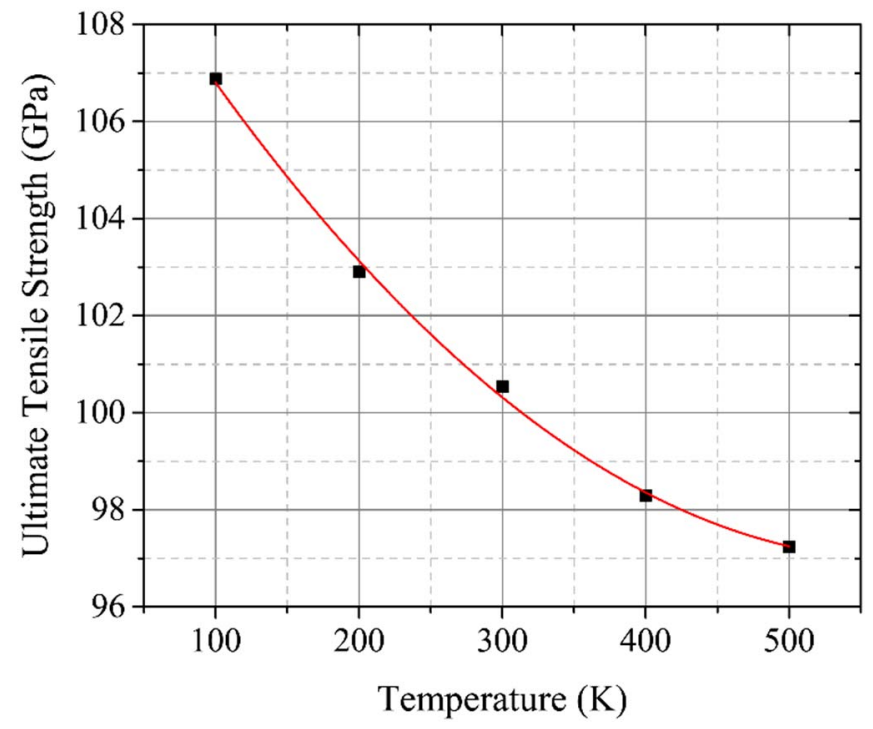

Fig. 4: Change of ultimate uniaxial tensile strength of $26 \mathrm{~nm} \times 5 \mathrm{~nm}$ armchair hBNNR with temperature
Ultimate uniaxial tensile strength drops from $106.87 \mathrm{GPa}$ at $100 \mathrm{~K}$ to $97.24 \mathrm{GPa}$ at $500 \mathrm{~K}$ and strain decreases from 0.33825 at $100 \mathrm{~K}$ to 0.31125 at $500 \mathrm{~K}$. Similar trend of ultimate strength and strain had been reported for single layer hexagonal boron nitride sheets [26] and graphene sheet. [27], [28]

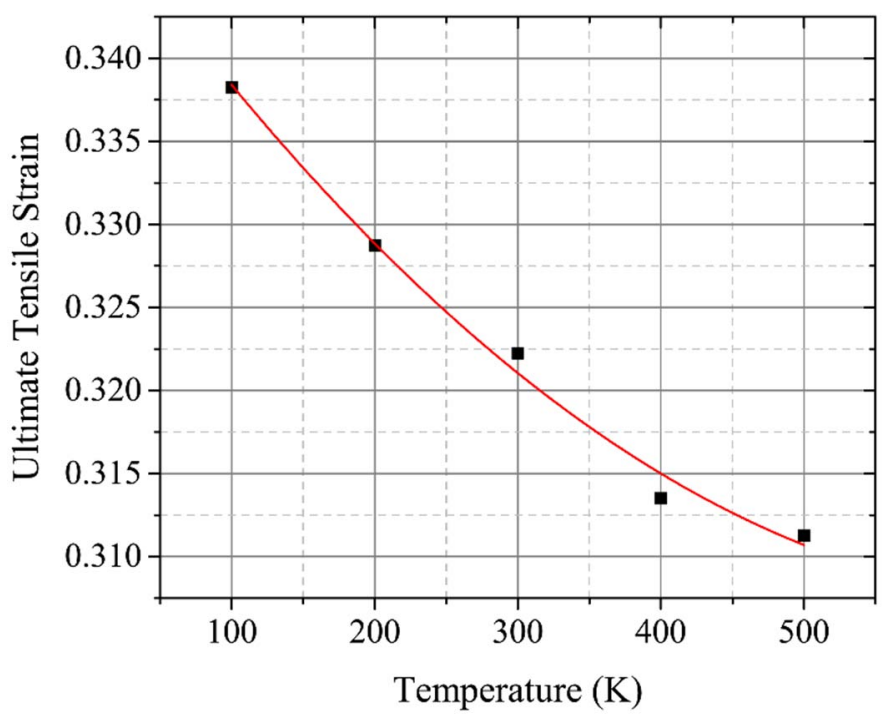

Fig. 5: Change of ultimate uniaxial tensile strain of $26 \mathrm{~nm} \times 5 \mathrm{~nm}$ armchair hBNNR with temperature

It is well known fact that change in strain rate effects the mechanical behavior of a material and hBNNR should be no exception. Change in stress-strain behavior is seen for change in strain rate. Increase in strain rate results in increase in ultimate uniaxial tensile strength and strain which is represented in Fig. 6.

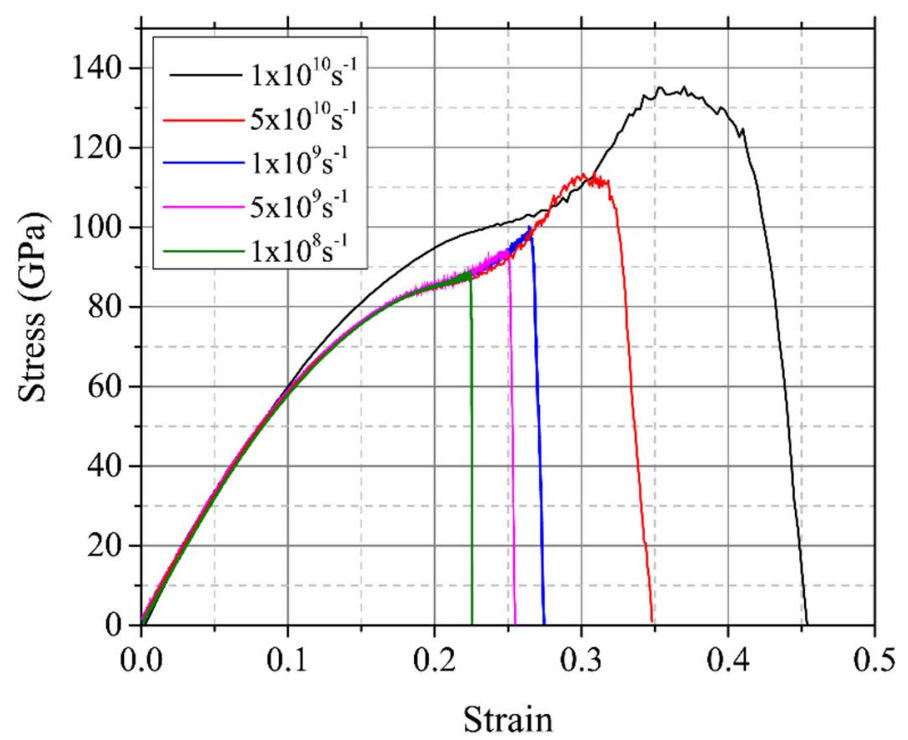

Fig. 6: Stress-strain curves of $26 \mathrm{~nm} \times 5 \mathrm{~nm}$ armchair hBNNR at different strain rates at a constant temperature of $300 \mathrm{~K}$ 
From Fig. 7 and Fig. 8, we can see that the ultimate uniaxial tensile strength and strain rises with increase in strain rate. $34.3 \%$ increase in ultimate uniaxial tensile strength and $42.8 \%$ increase in ultimate uniaxial tensile strain is observed for increase of strain rate from $1 \times 10^{8} \mathrm{~s}^{-1}$ to $1 \times 10^{10} \mathrm{~s}^{-1}$.

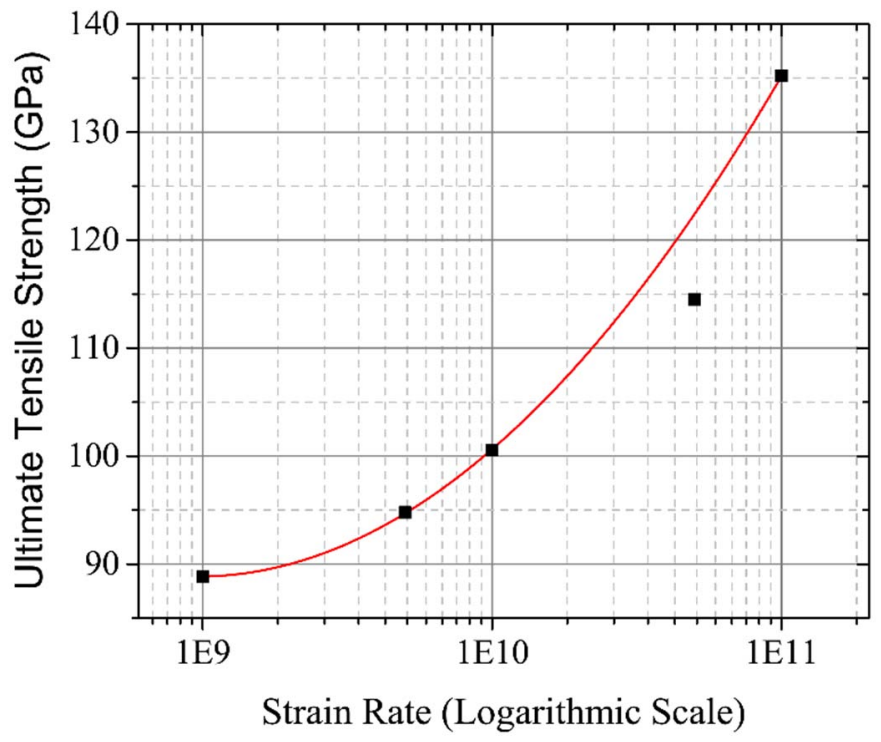

Fig. 7: Change of ultimate uniaxial tensile strength of $26 \mathrm{~nm} \times 5 \mathrm{~nm}$ armchair hBNNR with strain rate

Similar trend in change of tensile properties for change in strain rate can be seen in previous study done on boron nitride sheets. [27]

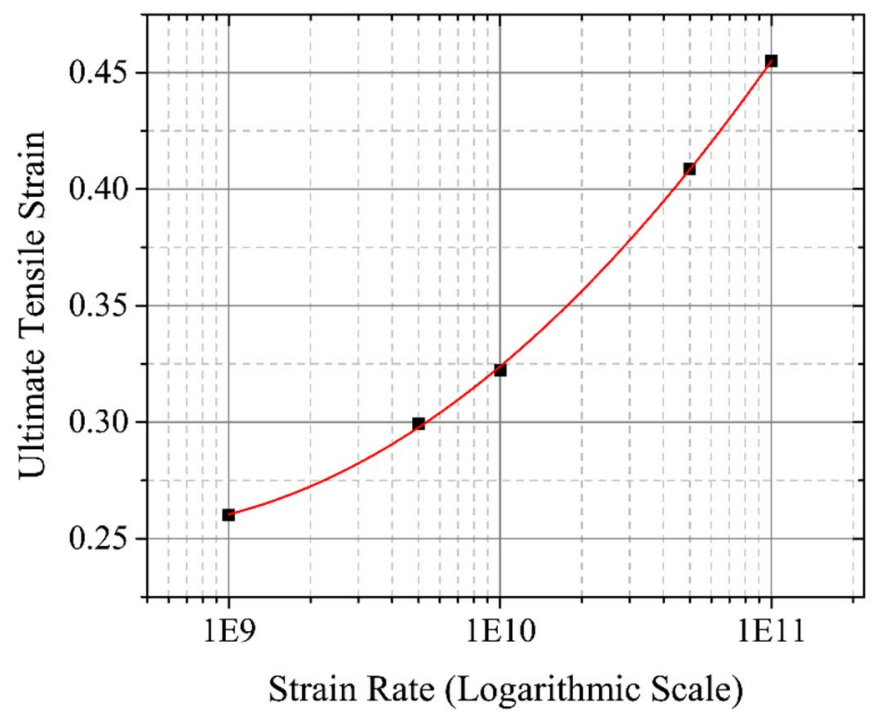

Fig. 8: Change of ultimate uniaxial tensile strain of $26 \mathrm{~nm} \times 5 \mathrm{~nm}$ armchair hBNNR with strain rate
Stress-strain curve of hBNNR structures with point vacancy and bi-vacancy has been shown in Fig. 9 and Fig. 10.

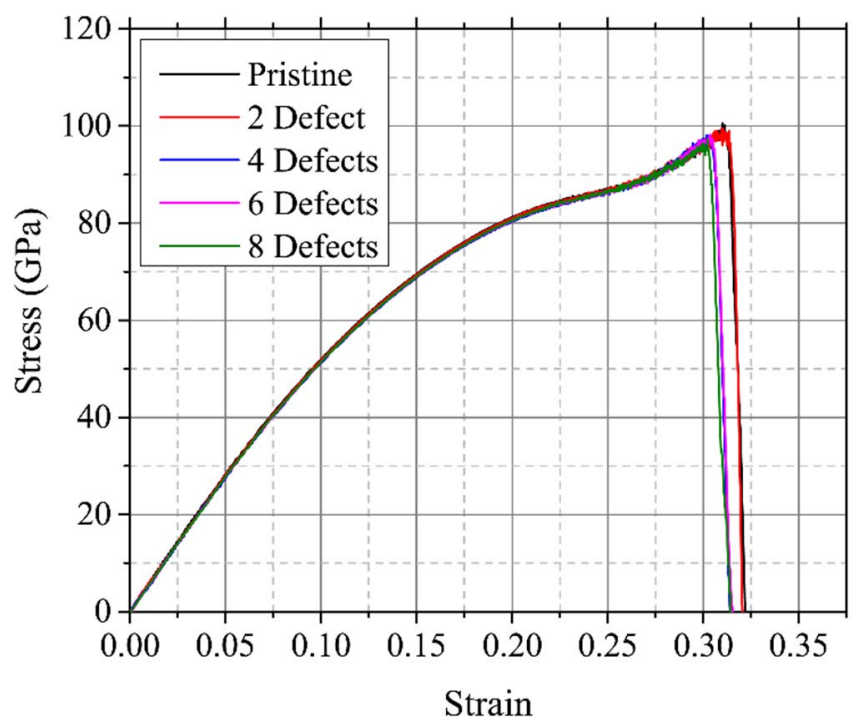

Fig. 9: Stress-strain curves of $26 \mathrm{~nm}$ x $5 \mathrm{~nm}$ armchair hBNNR with different amount of point vacancy

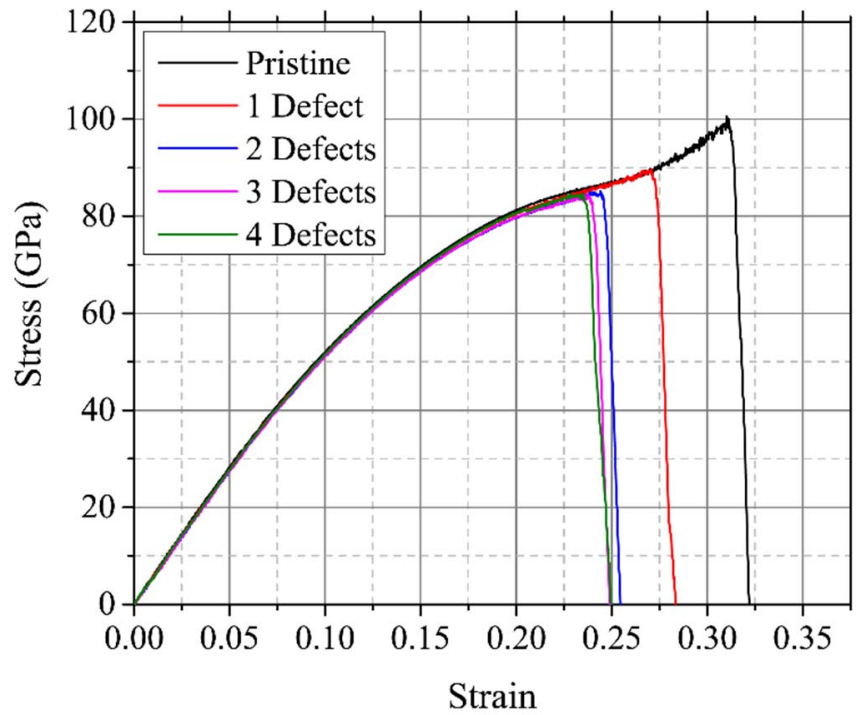

Fig. 10: Stress-strain curves of $26 \mathrm{~nm} \times 5 \mathrm{~nm}$ armchair hBNNR with different amount of bi-vacancy

Impact of point vacancy and bi-vacancy on tensile properties of hBNNR is shown in Fig. 11 and Fig. 12. Increasing defect causes decrease in ultimate uniaxial tensile strength and strain in hBNNR. We introduced these two types of defects keeping the number of atoms removed from the structure constant. Therefore, tensile simulation results acquired form the structure with two point vacancies were compared with the one with one bi-vacancy. 


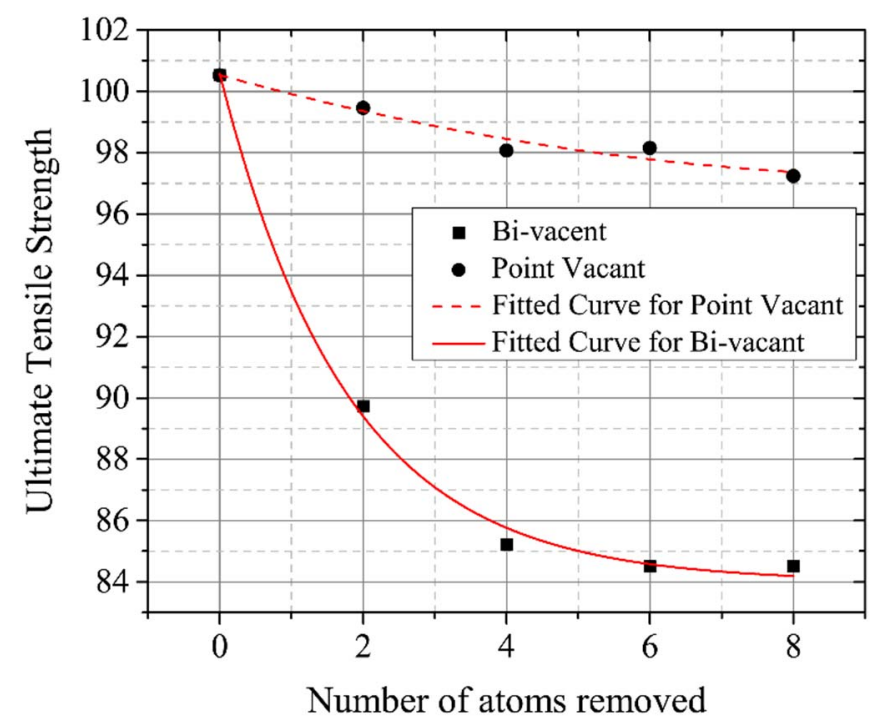

Fig. 11: Change of ultimate uniaxial tensile strength of $26 \mathrm{~nm} \times 5 \mathrm{~nm}$ armchair hBNNR with inclusion of vacancy defects

The effect of bi-vacancy if more prominent on the tensile properties of hBNNR compared to the effects of point vacancy. There is only $3.2 \%$ decrease in ultimate uniaxial tensile strength for the removal of eight atoms as point vacancy where the decrease is $15.9 \%$ for removal of same number of atoms as bi-vacancy. Ultimate uniaxial tensile strain for the removal of similar number of atoms decreased $2.5 \%$ for point vacancy and $22.4 \%$ for bi-vacancy.

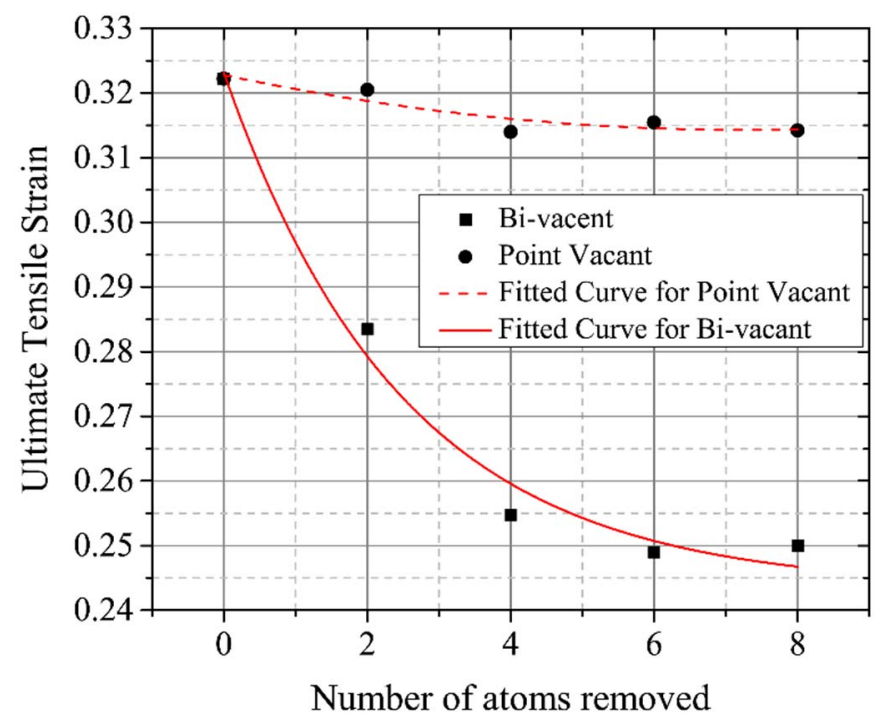

Fig. 12: Change of ultimate uniaxial tensile strain of $26 \mathrm{~nm} \times 5 \mathrm{~nm}$ armchair hBNNR with inclusion of vacancy defects

Fracture patterns during the tensile test of pristine hBNNR and defected hBNNR are presented in Fig. 13. The fracture of hBNNR with bi-vacancy clearly shows the initiation and propagation of fracture from the defect. But although structure with point vacancy shows decreased tensile strength and strain, the fracture did not start at any vacancy. Hence, the effect of bi-vacancies is greater on the tensile properties of hBNNR than point vacancies.
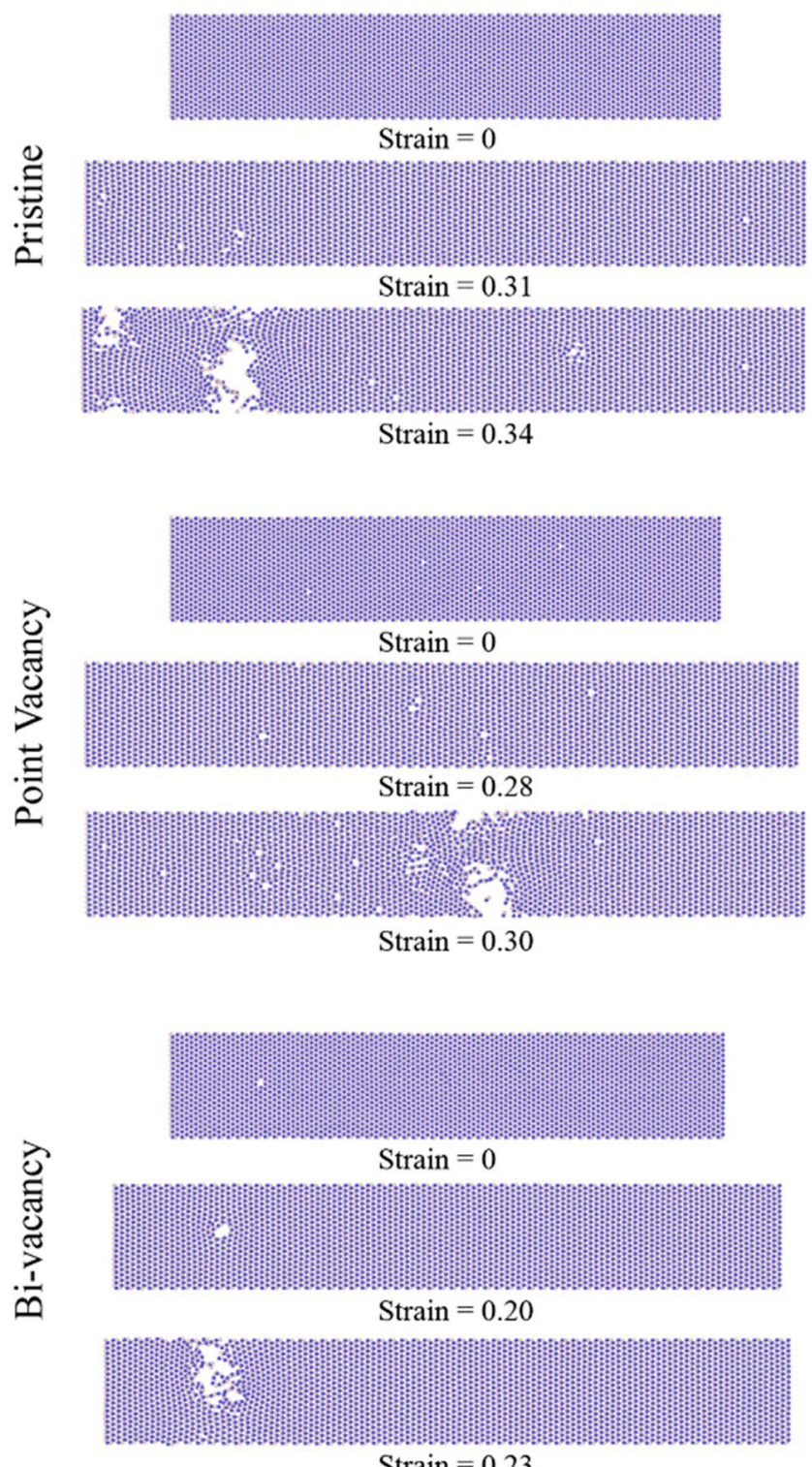

Fig. 13: Fracture patterns of hBNNR in pristine condition, with point vacancy and with bi-vacancy when uniaxial tensile strain is applied along horizontal direction ( $\mathrm{X}$ axis)

\section{CONCLUSION}

To conclude, the uniaxial tensile properties have been explored for pristine hBNNR at different temperatures, strain rates and after inclusion of defects. It is found that, ultimate tensile strength and ultimate tensile strain decrease with increasing temperature and increase with increasing strain rate. 
Ultimate tensile strength and strain decreased after we introduced vacancy defects in the hBNNR structure. The effect of bi-vacancy is observed to be more prominent on the tensile properties of hBNNR compared to point vacancy.

\section{REFERENCES}

[1] G. Cassabois, P. Valvin, and B. Gil, "Hexagonal boron nitride is an indirect bandgap semiconductor," Nat. Photonics, no. January, 2016.

[2] A. I. Khan, I. A. Navid, M. Noshin, H. M. A. Uddin, F. F. Hossain, and S. Subrina, "Equilibrium Molecular Dynamics (MD)

Simulation Study of Thermal Conductivity of Graphene

Nanoribbon: A Comparative Study on MD Potentials," Electronics, vol. 4, pp. 1109-1124, 2015.

[3] J. Zheng et al., "High thermal conductivity of hexagonal boron nitride laminates," 2D Mater., vol. 3, no. 1, pp. 1-6, 2016.

[4] L. Boldrin, F. Scarpa, R. Chowdhury, and S. Adhikari, "Effective mechanical properties of hexagonal boron nitride nanosheets," Nanotechnology, vol. 505702, p. 7, 2011.

[5] J. Yin et al., "Boron Nitride Nanostructures : Fabrication, Functionalization and Applications," Small, vol. 12, no. 22, pp. 2942-2968, 2016.

[6] T. Educational, "Mechanical Characterization of Boron-Nitride Nanoribbons via Nonlinear Structural Mechanics Georgios I. Giannopoulos 1," J. Nano Res., vol. 40, no. Md, pp. 58-71, 2016.

[7] J. Wu, B. Wang, Y. Wei, R. Yang, and M. Dresselhaus, "Mechanics and Tunable Bandgap by Straining in Single-Layer Hexagonal Boron-Nitride," Mater. Sci.,pp. 1-16, 2013.

[8] S. Seetharaman, J. Subramanian, K. S. Tun, A. S. Hamouda, and M. Gupta, "Synthesis and Characterization of Nano Boron Nitride Reinforced Magnesium Composites Produced by the Microwave Sintering Method," Materials (Basel)., vol. 6, pp. 1940-1955, 2013.

[9] P. D. G. Kvashnin, M. Ghorbani-Asl, D. V. Shtansky, D. Golberg, A. V. Krasheninnikov, Sorokin and B., "Mechanical properties and current-carrying capacity of $\mathrm{Al}$ reinforced with graphene/BN nanoribbons: a computational study," Nanoscale, vol. 0, pp. 1-3, 2013.

[10] M. Le, "Size effects in mechanical properties of boron nitride nanoribbons †," J. Mech. Sci. Technol., vol. 28, no. 10, pp. 4173 $4178,2014$.

[11] B. Nitride et al., “'White Graphenes': Boron Nitride Nanoribbons via Boron Nitride Nanotube Unwrapping," Nano Lett., vol. 10, pp. 5049-5055, 2010.

[12] J. Yin et al., "Large Single-Crystal Hexagonal Boron Nitride Monolayer Domains with Controlled Morphology and Straight Merging Boundaries," Small, vol. 11, no. 35, pp. 4497-4502, 2015.

[13] N. Ding, X. Chen, and C. L. Wu, "Mechanical properties and failure behaviors of the interface of hybrid graphene / hexagonal boron nitride sheets," Nat. Publ. Gr., vol. 6, no. May, pp. 1-9, 2016.

[14] M. Griebel, J. Hamaekers, and F. Heber, "A molecular dynamics study on the impact of defects and functionalization on the Young modulus of boron - nitride nanotubes," Comput. Mater. Sci., vol. 45, no. 4, pp. 1097-1103, 2009.

[15] D. H. Kim, H. S. Kim, M. W. Song, S. Lee, and S. Y. Lee, "Geometric and electronic structures of monolayer hexagonal boron nitride with multi vacancy," Nano Converg., pp. 0-7, 2017.

[16] A. Sinitskii et al., "High-Yield Synthesis of Boron Nitride Nanoribbons via Longitudinal Splitting of Boron Nitride Nanotubes by Potassium Vapor," Am. Chem. Soc., no. 10, pp. 9867-9873, 2014.

[17] B. Mortazavi, "Thermal conductivity and tensile response of defective graphene: A molecular dynamics study," Carbon N. Y., vol. 63, pp. 460-470, 2013.

[18] A. I. Khan, R. Paul, and S. Subrina, "Thermal transport in graphene/stanene heterobilayer nanostructures with vacancies: an equilibrium molecular dynamics study," RSC $A d v$., vol. 7, pp. 44780-44787, 2017

[19] S. Plimpton, "Fast Parallel Algorithms for Short - Range Molecular Dynamics," J. Comput. Phys., vol. 117, no. June 1994, pp. 1-19, 1995.

[20] L. Lindsay and D. A. Broido, "Enhanced thermal conductivity and isotope effect in single-layer hexagonal boron nitride," Phys. Rev. $B$, vol. 84, no. 15, p. $155421,2011$.

[21] B. Mortazavi and Y. Rémond, "Investigation of tensile response and thermal conductivity of boron-nitride nanosheets using molecular dynamics simulations," Phys. E Low-Dimensional Syst. Nanostructures, vol. 44, no. 9, pp. 1846-1852, 2012.

[22] G. Rajasekaran, P. Narayanan, and A. Parashar, "Effect of Point and Line Defects on Mechanical and Thermal Properties of Graphene: A Review," Crit. Rev. Solid State Mater. Sci., vol. 8436, no. October, pp. 1-25, 2015.

[23] M. Noshin, A. I. Khan, I. A. Navid, H. M. A. Uddin, and S. Subrina, "Impact of vacancies on the thermal conductivity of graphene nanoribbons : A molecular dynamics simulation study" AIP $A d v$., vol. 7, 2017.

[24] J. H. Warner, M. H. Rümmeli, A. Bachmatiuk, and B. Büchner, "Atomic resolution imaging and topography of boron nitride sheets produced by chemical exfoliation," ACS Nano, vol. 4, no. 3, pp. 1299-1304, 2010.

[25] D. E. Smith and A. D. J. Haymet, "Free energy, entropy, and internal energy of hydrophobic interactions: Computer simulations," J. Chem. Phys., vol. 98, no. 8, pp. 6445-6454, 1993.

[26] T. Han, Y. Luo, and C. Wang, "Effects of temperature and strain rate on the mechanical properties of hexagonal boron nitride nanosheets," J. Phys. D. Appl. Phys., vol. 47, no. 2, p. 25303, 2014 T. FANG, W. CHANG, and J. Yang, "Temperature Effect on Mechanical Properties of Graphene Sheets Under Tensile Loading," Dig. J. Nanomater. ..., vol. 7, no. 4, pp. 1811-1816, 2012.

[28] T. Han, P. He, and B. Zheng, "Dependence of the tensile behaviour of single graphene sheet on temperature and strain rate," pp. 1-2. 